\title{
Peridynamic Simulation of Fatigue Crack Growth Behaviour with the Effect of Microstructure
}

\author{
Binchao Liu ${ }^{1}$, Rui Bao ${ }^{1, *}$, Yamei Niu ${ }^{1}$, Songsong Lu${ }^{1}$, and Kai Wang ${ }^{1}$ \\ ${ }^{1}$ Institute of Solid Mechanics, Beihang University, China
}

\begin{abstract}
The purpose of this paper is to explore the influences of microstructures on crack growth behaviour in 2324-T39 aluminum alloy based on peridynamic(PD) theory. The microelastic bond-based peridynamic constitutive is modified as microplastic to describe the plasticity of aluminum alloys. A new method to establish polycrystalline models based on metallographs is adopted, and grains are reflected in simulations by setting transgranular and intergranular pairwise force in the corresponding bonds. Two kinds of microstructures are modeled according to metallographs, and a special kind of crack branch resulted from the link-up of the secondary crack with the main crack and the growth of the branched crack is successfully captured. The PD simulations reveal that microstructure orientation characteristics have an impact on crack propagation paths and crack growth modes, and it is easier for the secondary-crack resulted macroscopic crack branching to appear if grain boundaries locate not too close to the leading crack tip but within the crack tip plastic zone. The numerical results are verified by experiments and fractographic analysis.
\end{abstract}

\section{Introduction}

According to achievements in fracture mechanics, a through-thickness middle crack in an isotropic thin plate subjected to remote tension-dominated loading should propagate perpendicular to the load direction, i.e. Mode I. Under the above conditions, however, phenomenon such as crack kinking, bifurcation and branching are observed in the experiments, and it is discovered that material microstructure is one of the affecting factors $[1$, 2]. For metals with small grains, the influence of microstructure is embodied in microscale and inconspicuous in macroscale; for relatively larger grains or grains with apparent orientation, however, its influence may result in the macroscale differences of crack growth behaviors. Such impacts are observed even in traditional rolled aluminum alloys if their microstructures exhibit apparent features. For instance, Bao [3] observed a link-up crack branching in AA2324T39 and AA7050-T7451, and $\mathrm{Lu} \mathrm{[4]} \mathrm{discovered} \mathrm{that} \mathrm{the}$ branching is affected by different microstructures of aluminum alloys.

Studying the crack growth behavior under the influence of microstructures has a great significance on damage tolerance design and evaluation of crack growth life. On one hand, researches on such impacts rely on experiments, which can manifest special phenomena and uncover general rules; on the other hand, numerical simulations based on experimental phenomena also play an important role with improving computing power and simulation technologies, for it largely reduces experiment costs as well as reveals key parameters under complex circumstances. Several methods such as cohesive zone model(CZM), extended finite element(XFEM) and molecular dynamics(MD), have been proposed to simulate crack growth and branching behaviour with varying success, but they suffer from various problems in modelling the fracture problems and the influence of material microstructure. For the finite element based methods, the fundamental difficulties lie in the assumption of classical continuum theory that the body remains continuous as it deforms. However, once a crack initiates and propagates, which means discontinuities appear, such assumption is violated. From the standpoint of mathematics, the classical continuum theory is formulated using spatial partial differential equations and these spatial derivatives lose their meanings at discontinuities where cracks occur [5]. For MD, however, time and spatial scale are limited to microscale, making it applicable to explain mechanism from molecular level but incompetent to predict crack growth behaviors in practical structures [6].

Peridynamics (PD), proposed by Silling in 2000 [7], has aroused intense scholarly interest since its birth and becomes a promising method to study crack propagation. It attempts to unify the mechanics of continuous and discontinuous media within one framework. The theory replaces the spatial derivatives with integrals that are valid regardless of the existence of a discontinuity, so that the peridynamic governing equations are applicable at fractures and external crack growth criteria are not necessary. Therefore, complex phenomena such as crack nucleation, crack branching, coalition of multiple cracks and crack arrest occur spontaneously, which sheds a new light on simulating crack growth in materials with complex orientation or microstructures [5].

\footnotetext{
Corresponding author: rbao@buaa.edu.cn
} 
In this paper, the original bond-based peridynamic (PD) theory is briefly introduced firstly. In order to simulate mechanic behaviors of aluminum alloy, the microelastic bond-based peridynamic constitutive is then extended to be microplastic. The models with artificial grains are established to make a proper difference in pairwise force between transgranular and intergranular bonds. Further, grain models according to metallographs of aluminum alloy (AA) 2324-T39 are established to observe crack growth behavior, and compared with test results.

\section{Peridynamic constitutive}

\subsection{Original PMB model in Peridynamics}

The original prototype microelastic brittle(PMB) material model, which is the first bond-based peridynamic model, was proposed by Silling in 2000 [7]. As shown in Fig. 1, assuming that an object occupies a certain spatial domain $R$ in the reference configuration at time $t$, there exists a pairwise force $f$ between one material point $x$ and any other material point $x^{\prime}$ located inside its peridynamic horizon $\delta$,

$$
f\left(x, x^{\prime}, u(x, t), u\left(x^{\prime}, t\right), t\right)
$$

where $u(x, t)$ is the displacement of material point $x$. As a contrast, there are no such interactions for material points out of the peridynamic horizon, which is similar to the theory of molecular dynamics.



Fig. 1. The diagrammatic drawing of pairwise bond force between material points in original PMB.

According to the Newton's Second Law, the peridynamic governing equation can be written as

$\rho(x) u(x, t)=\int_{H} f\left(x, x^{\prime}, u(x, t), u\left(x^{\prime}, t\right), t\right) d V^{\prime}+b(x, t)$

where $\rho(\mathrm{x})$ denotes the material density, $b(x, t)$ denotes a prescribed body force, and $H$ denotes the neighborhood of material point $x$,

$$
H=H(x, t)=\left\{x^{\prime} \in R,\left\|x^{\prime}-x\right\| \leq \delta\right\}
$$

In the following discussion, the relative position of two material points in the reference configuration is denoted as $\xi$, and their relative displacement is denoted as $\eta$,

$$
\xi=x^{\prime}-x, \eta=u\left(x^{\prime}, t\right)-u(x, t)
$$

thus $\xi+\eta$ denotes current relative position between the particles.

It can be seen that all the mechanic responses are included in the peridynamic bond force $f$, which is also the key in establishing peridynamic models. In the bondbased peridynamics, pairwise force between two material points in elastic materials can be expressed as

$$
f=\operatorname{cs} \mu
$$

where bond constant $c$ is the peridynamic material parameter,

$$
c=\frac{18 K}{\pi \delta^{4}}
$$

where $K$ denotes the bulk modulus and $S$ the relative stretch defined as

$$
s=\frac{|\xi+\eta|-|\xi|}{\xi}
$$

and $\mu$ is a parameter to judge whether the bond connecting two particles is broken,

$$
\mu(x, \xi, t)=\left\{\begin{array}{l}
1, s<s_{0} \\
0, s>s_{0}
\end{array}\right.
$$

where $S_{0}$ denotes critical stretch. When relative stretch exceeds the critical stretch $s_{0}$, the bond is broken and two particles no longer interact with each other. Further on, the damage of material point $x$ is defined as the broken bonds' proportion of all bonds connected to material point $x$,

$$
\varphi(x, t)=1-\frac{\int_{H} \mu(x, \xi, t) d V_{\xi}}{\int_{H} d V_{\xi}}
$$

when it reachs certain value $\varphi_{0}$, it is regarded that crack occurs on this matrial points.

\subsection{Constitutive extension}

So far only elastic materials have been discussed for the bond-based model as shown in Fig. 2(a). However, many metal materials like aluminum alloy display strong and apparent plastic behavior in mechanical response, thus plasticity must be taken into consideration in peridynamic constitutive establishment. Fig. 2 (b) shows that plasticity can be incorporated into microelastic bond-based model by treating each bond as though it has its own yield stress [8]. This bond-based material model is called microplastic, and the yield force density $f_{\text {yield }}$ or the yield stretch $S_{\text {yield }}$ can be calibrated according to the measured material yield stress. In order to simulate 
elastoplastic mechanic behaviors of aluminum alloy, this microplastic bond-based peridynamic model is adopted in this paper.

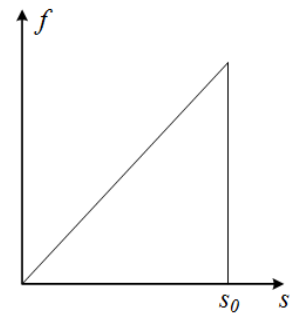

(a)

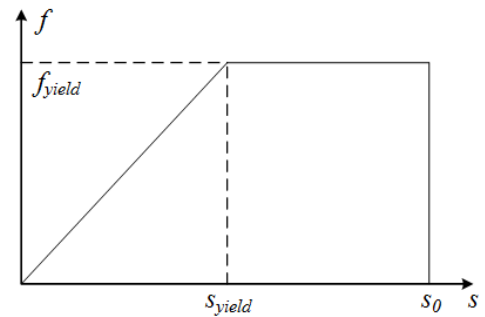

(b)
Fig. 2. The micro-elastic PMB model; (b) The micro-plastic model of extended PMB.



Fig. 3. Computation of force per unit area from bond force densities.

Assuming conditions of uniaxial strain in $x 1$ direction shown in Fig. 3, the yield force density can be calibrated to the material yield stress as

$\sigma_{y}=2 \pi \int_{0}^{\delta} \int_{0}^{\xi} \int_{0}^{\cos ^{-1} \frac{z}{\xi}}\left(f_{\text {yield }} \cos \phi\right) \xi^{2} \sin \phi d \phi d z d \xi=\frac{\pi \delta^{4} f_{\text {yield }}}{6}$

where $\sigma_{y}$ is the normal stress in uniaxial strain after all the bonds have yielded. However, it is obvious that bonds do not all yield at the same value of bulk strain, for bonds in $x_{1}$ direction are bound to yield earlier than others, and the initial yield normal stress $\sigma_{0}$ can be derived similarly as

$$
\sigma_{0}=\frac{\pi c \delta^{4} \varepsilon_{11}}{10}
$$

where $\varepsilon_{11}$ is the bulk normal strain component. On the other hand, this material starts to yield when $c s=f_{\text {yield }}$ , hence

$$
\varepsilon_{11}=\frac{f_{\text {yield }}}{c}
$$

thus we arrive at

$$
\sigma_{0}=\frac{3 \sigma_{y}}{5}
$$

which indicates that the material displays elastic response before the normal stress reaches initial yield stress $\sigma_{0}$, and then experiences a strain hardening response until all bonds yield. Such response is similar to the stress-strain curve of aluminum alloys. The yield stretch is finally obtained by combining (6), (11) and (13):

$$
s_{\text {yield }}=\frac{\sigma_{y}}{3 K}
$$

The stress-strain curve given by updated microplastic PMB is shown as red dash line in Fig. 4. Compared with the blue solid line given by primer microelastic PMB, the updated PMB constitutive displays obvious yielding process, which is to some extent consistent with experiment data of AA2324-T39. Therefore, the improved constitutive is suitable to describe the mechanical response of aluminum alloy.

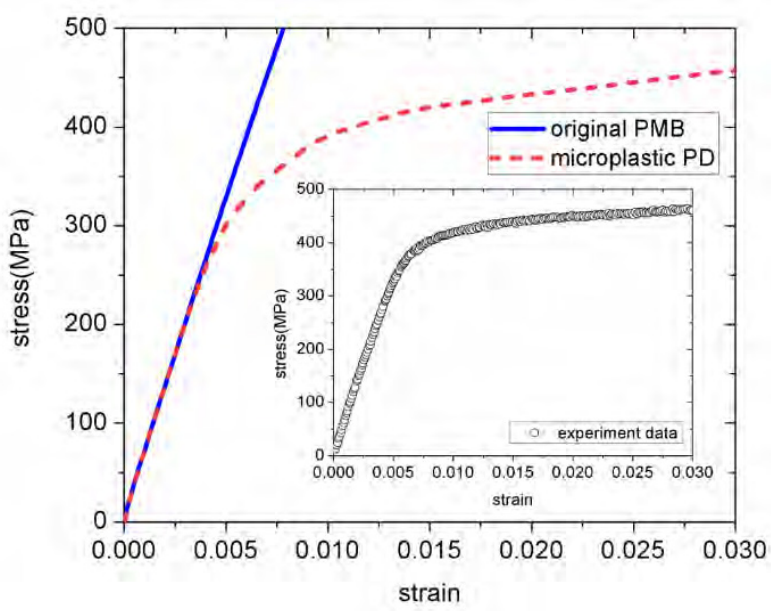

Fig. 4. Compared with primer microelastic PMB, the stressstrain curve of updated microplastic PMB gives obvious yielding process, which is more consistent with experiment data and suitable to describe mechanical response of aluminum alloy.

\section{Models and parameters}

In many studies concerning material microstructure, Voronoi tessellation method is adopted to establish polycrystalline models. In this study, however, a new modeling method using metallographs is adopted to exactly reflect the unique grain characteristics in materials. Metallograph of AA2324-T39 is respectively shown in Fig. 5(a), from which can be seen that AA2324-T39 microstructure displays apparent directional feature with its size in longitudinal orientation (L-orientation) larger than transverse orientation (T-orientation). According to different gray level in different region in metallographs, grains can be distinguished. Then we paint grains in different colors and obtain a picture with grains distinguished by a color characteristic value, RGB for example, as shown in Fig. 5 (b). The picture is afterwards imported into MATLAB for discretization and the spacial coordinates of the 
particles can be obtained, during which the picture magnification as well as pixel size must be taken into consideration. Meanwhile, the RGB map of picture is also read by MATLAB. If the RGB values of two particles equal with each other, the bond connecting them is of transgranular type and its pairwise force is computed as $f_{\text {trans }}$; otherwise, the bond is of intergranular type and its pairwise force is computed as $f_{\text {inter }}$.

Following the methods above, two plates with different microstructures according to the metallograph, namely L-T oriented AA2324-T39 and T-L oriented AA2324-T39, are established in the simulations as shown in Fig. 5 (c) and (d). The length, width and thickness of the model are respectively $5 \mathrm{~mm}, 2.25 \mathrm{~mm}$ and $150 \mu \mathrm{m}$, with a pre-crack of $0.8 \mathrm{~mm}$. The spacing between material points in PD is set as $25 \mu \mathrm{m}$, which is considered to be small enough in comparison to the average grain size. To take the different transgranular and intergranular interaction into consideration, bond force in transgranular bond and intergranular bond is set different. The transgranular bond force is computed according to Eq. (6), and $f_{\text {inter }} / f_{\text {trans }}=0.5$ is adopted.



(a)

(b)

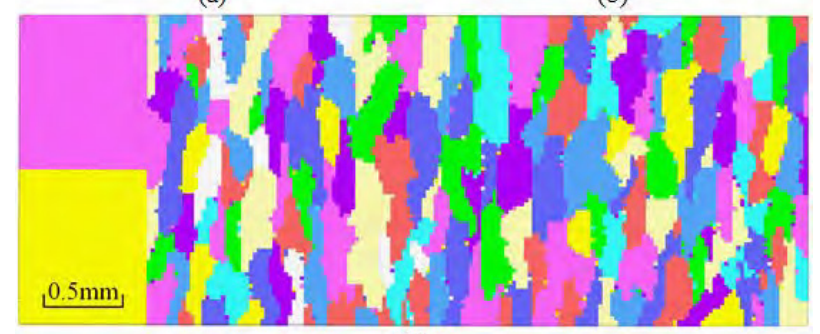

(c)

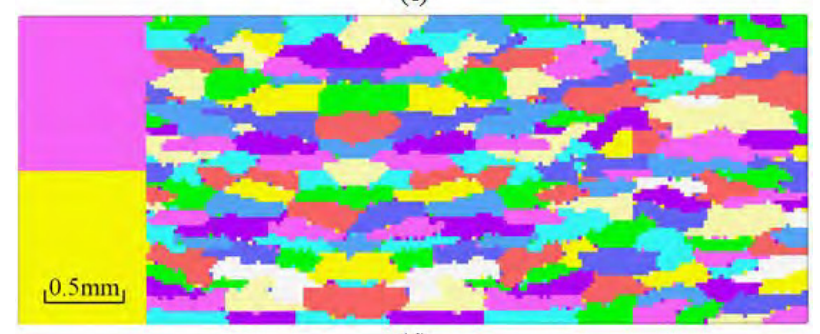

(d)

Fig. 5. (a) The metallograph of AA2324-T39; (b) the picture with painted grains; (c) the model with metallograph grains of L-T oriented AA2324-T39 and (d) the model with metallograph grains of T-L oriented AA2324-T39.

Numerical simulations are conducted by the perimodule of Large-scale Atomic Molecular Massively Parallel Simulation (PDLAMMPS), and material parameters are inputted according to mechanical properties of AA2324-T39 obtained from previous experiments [9], where the Young's modulus is $E=74 \mathrm{GPa}$, the material density is $\rho=2730 \mathrm{~kg}^{\prime} \mathrm{m}^{-3}$, the ultimate strength is $\sigma_{\mathrm{b}}=530 \mathrm{MPa}$ and the yield strength is $\sigma_{0.2}=480 \mathrm{MPa}$. Here, the slight distinctions between elastic modulus observed in experiments of L-T oriented AA2324-T39 and T-L oriented AA2324-T39 are ignored. Considering both the computational accuracy, stability and convergence requirement, the horizon is set as 3.03 times the spacing of PD material points, where accordingly the critical damage value is about $\varphi_{0}=0.35$. The timestep is set as $2 \times 10^{-9} \mathrm{~s}$, which is small enough for the computational stability. To be consistent with the Kmax-level in the experiments, a remote tension stress of $150 \mathrm{MPa}$ is applied. The yield stretch is set as 0.0036 according to Eq. (14), and the critical stretch is set as 0.0065 .

\section{Branching in crack paths}

\subsection{Branching in crack paths}

Crack growth processes of L-T oriented AA2324-T39 are shown in Fig. 6, which indicate that the appearance of secondary crack, the link-up of the secondary crack with the main crack and the growth of the branched crack can be successfully captured in PD simulations. The crack trajectory and the branching process agree quite well with those observed in the experiments shown in Fig. 7, and one obvious change of crack growth rate in branching process is marked by rectangle in Fig. 9(a). The whole branching process can be summarized and explained as follows:

(1) Firstly, before the secondary crack appears, the leading crack tip front is obstructed by a grain boundary perpendicular to the direction of crack propagation (Fig. 6, 116000 steps; Fig. 9(a), point A). The crack slows down or even stops growing because the bonding cohesion inside grains is stronger than the grains boundary so that crack needs more energy to grow through the grain.

(2) Secondly, the secondary crack appears on the boundary nearby the crack tip (Fig. 6, 177000 steps; Fig. 9(a), point B). The cohesion between grains is relatively weaker than inside, so it is easier for damage to accumulate here and thus for secondary crack to generate.

(3) At last, as the secondary crack grows, it is connected to the leading crack along the grain boundary which prevents the growth of leading crack (Fig. 6, 228000 steps; Fig. 9(a), point C). 


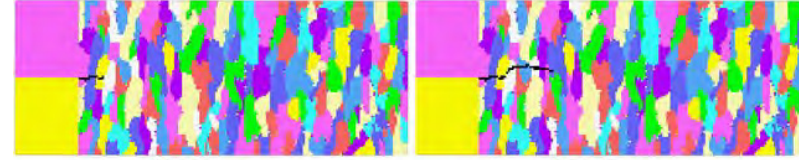

63000 steps

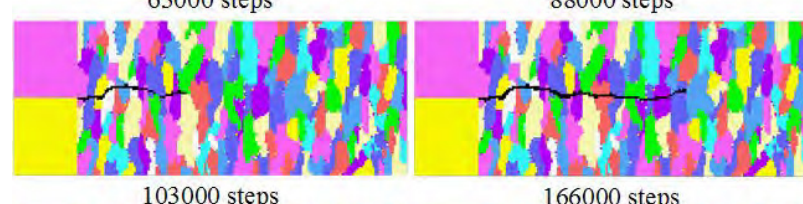

103000 steps

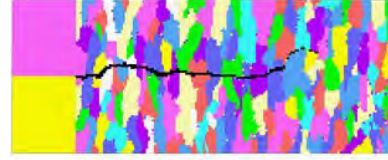

177000 steps
166000 steps



228000 steps

Fig. 6. The PD simulation of crack growth process in L-T oriented AA2324-T39.



(a)

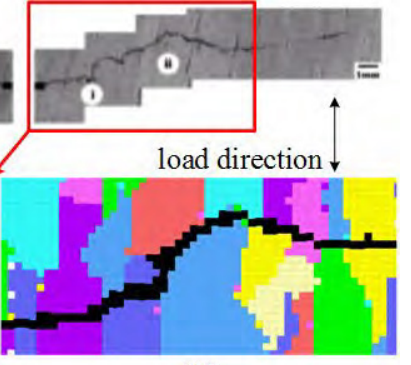

(b)

Fig. 7. (a) The experimental results in [4] and (b) the PD simulation results of crack propagation path in L-T oriented AA2324T39 which is a part of FIG. 6.

Crack growth processes of T-L oriented AA2324T39 are shown in Fig. 8, which are similar to those of LT orientated AA2324-T39. However, the location of the secondary cracks is much nearer to the main crack tip, which leads to smaller deflection of the whole crack path. The reason is that the secondary crack appears on grain boundaries and the dimension of the grains in $\mathrm{T}$ direction is relatively smaller than that in $\mathrm{L}$ direction. This phenomenon matches the experimental observation.

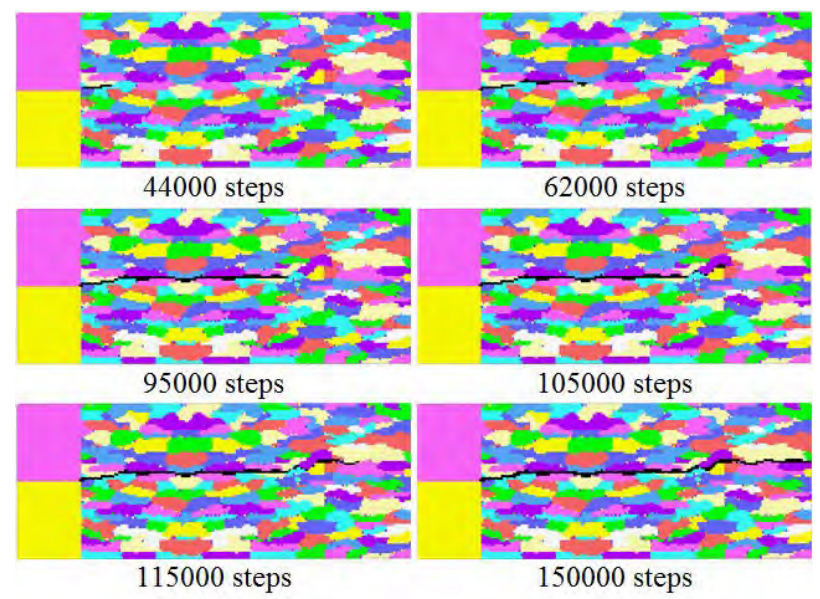

Fig. 8. The PD simulation of crack growth process in T-L oriented AA2324-T39.

It is worth noting that there are also some differences between the crack growth mode of L-T and T-L oriented AA2324-T39. For the L-T orientation, the leading crack

tends to display transgranular propagation in most cases, and only display intergranular propagation when the secondary crack just appears. For the T-L orientation, the crack always displays intergranular propagation. Moreover, the observation that crack propagates faster in T-L oriented AA2324-T39 than L-T oriented AA2324T39, which is shown in Fig. 9(c), accords well with the experiment results shown in Fig. 9(d). In fact, transgranular propagation mode needs and consumes more energy, and it is natural that cracks grow slower than intergranular propagation mode. Thus the differences of propagation mode and growth rate complement each other. The black rectangles in Fig. 9(a) indicates the moments when obvious crack branches occur. 


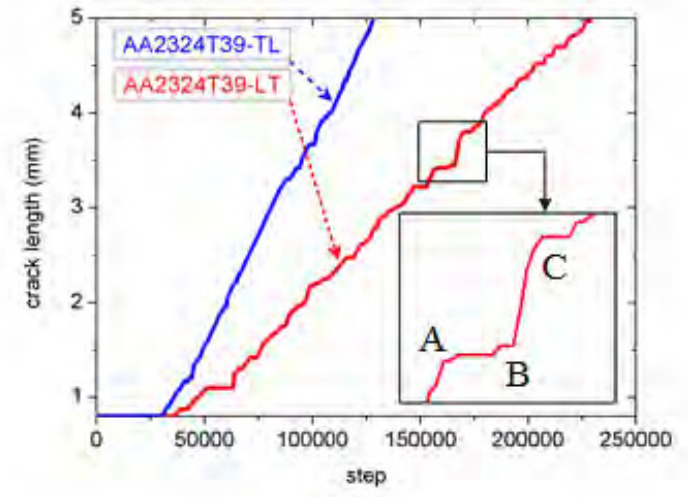

(a)
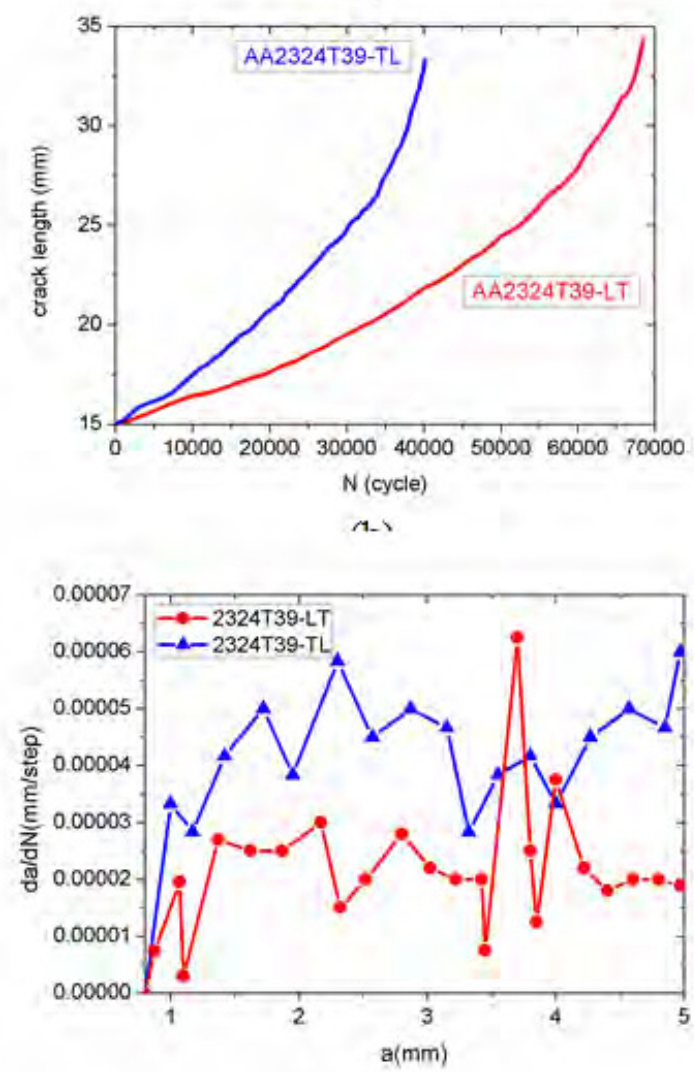

(c)

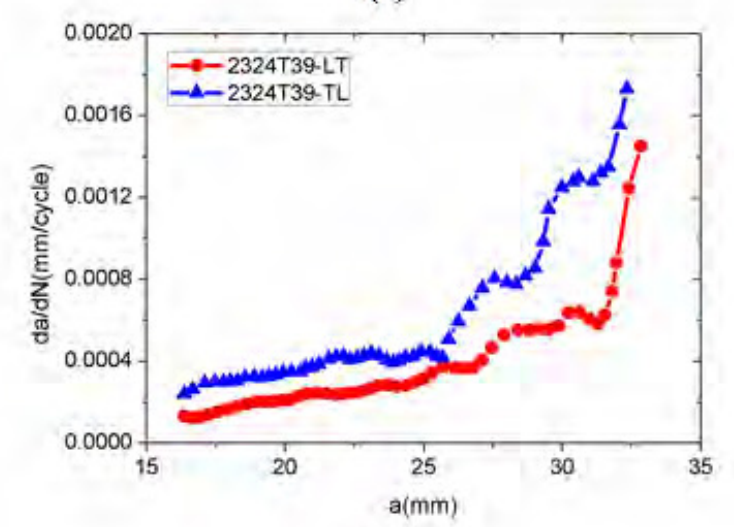

(d)

Fig. 9. The crack length obtained from (a) PD simulations and (b) experiments; and the crack growth rate obtained from (c) PD simulations and (d) experiments.

\subsection{Secondary crack location}

The mechanism of secondary crack has been studied through fracture surface analysis and finite element simulations in previous studies [4,10]. It has been pointed out the surface secondary crack which results in macroscopic crack branching appears within the leading crack plastic zone.

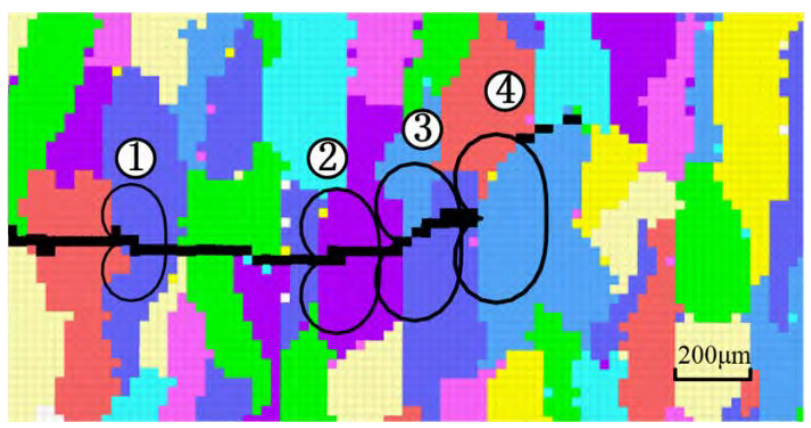

Fig. 10. The relationship of the crack tip plastic zone and the location of the second crack.

As mentioned above, the crack branching similar to the experimental results has been observed in PD simulations, and the relationship between the position of secondary crack and crack tip plastic zone is verified in PD as follows. Four corresponding plastic zones of leading crack are marked in Fig. 10, including two place (3) (4) where intergranular crack appear and other two places (1)(2) with no intergranular cracks. The plastic zones of leading crack in plane stress cases are estimated by Eq. (15), which is the curve equation of the crack tip plasticity boundaries in the condition of small scale yielding under Mises criterion. It can be concluded from Fig. 10 that the surface secondary crack appears along the grain boundary within the scope of leading crack plastic zone, as marked by (3) and (4). Under the circumstances where there are no grain boundaries located in the crack tip plastic zone of the leading crack, as marked by (1) and (2), it is rare for the secondary cracks to initiate.

$$
r_{p}=\frac{K_{\mathrm{I}}^{2}}{2 \pi \sigma_{0.2}^{2}} \cos ^{2} \frac{\theta}{2}\left[1+3 \sin ^{2} \frac{\theta}{2}\right]
$$

It is widely acknowledged that the deformation of material is relatively larger in the crack tip plastic area, thus the damage resulted from dislocations is also larger, and the secondary crack is easier to initiate at weaker points such as holes or inclusions. In PD simulations, the grain boundary is set to be relatively weak, so the secondary crack is inclined to appear on the grain boundary alongside the propagation direction in crack tip plastic area. Further, it is of greater probabilities for the link-up resulted crack branching to appear when the grain boundary locates from proper distances to the crack tip in plastic area. If the grain boundary is too close to the crack tip, the secondary crack on the grain boundary will be directly connected to the leading crack, and the crack branching is not obvious to observe. If there are no grain boundaries located in the crack tip plastic zone, on the contrary, it is likely for the leading 
crack to propagate transgranularly and therefore of little probabilities for the secondary crack to appear.

\section{Conclusions}

According to the PD simulation in effects of grain size and orientations on crack paths, it can be concluded that:

(1) The crack branching process and crack path trajectories in AA2324-T39 resulted from the linkup of secondary crack with the main crack can be reasonably captures by PD method.

(2) The crack path in AA2324-T39 can be reasonably described in PD simulations by setting distinct pairwise force in transgranular and intergranular bonds.

(3) According to the PD simulations, the whole crack path in L-T oriented AA2324-T39 is more circuitous than that observed in T-L oriented AA2324-T39. These results indicate that the microstructure grain size and orientation characteristics has an influence on the crack growth paths.

(4) In PD simulations, it is observed in L-T orientated AA2324-T39 model that the leading crack displays generally transgranular propagation, and the secondary crack which appears on the grain boundary usually displays intergranular propagation. As for T-L oriented AA2324-T39, however, the cracks always display intergranular propagation. The results indicate that the microstructure grain size and orientation characteristics also has an impact on the crack growth mode.

(5) It is easier for the secondary-crack resulted macroscopic crack branching to appear if grain boundaries locate not too close to the leading crack tip but within the crack tip plastic zone.

\section{References}

1. J. Toribio, B. González, J.C. Matos, Eng. Fract. Mech. 77, 11 (2010).

2. M. Benedetti, M. Beghini, V. Fontanari, B. Monelli, Int. J. Fatigue, 3111 (2009)

3. R. Bao, X. Zhang, Int. J. Fatigue, 327 (2010)

4. S. Lu, R. Bao, T.Zhang, B. Fei,. Int. J. Fatigue, 92, 459-469, (2016)

5. A. Agwai, I. Guven, E. Madenci, Int. J. Fract., 1711 (2011)

6. B. N. Cox, H. Gao, D. Gross, D. Rittel, J. Mech. Phys. Solids, 5332005.

7. S. A. Silling, J. Mech. Phys. Solids, 481 (2000)

8. F. Bobaru, J. T. Foster, P. H. Geubelle, S. A. Silling,. Handbook of peridynamic modeling ( $\mathrm{Crc}$ Press, Boca Raton, 2016)

9. T. Zhang, B. Rui, B. Fei, Int. J. Fatigue, 581 (2014)

10. T. Zhang, B. Rui, S. S. Lu, B. Fei, Int. J. Fatigue, 82, 602-615, (2016) 